\title{
Using an Off-the-Shelf Lock-In Detector for Admittance Spectroscopy in the Study of Plants
}

\author{
Fábio D. R. Monteiro, Peter Stallinga \\ University of The Algarve, Faro, Portugal \\ Email: peter.stallinga@gmail.com
}

How to cite this paper: Monteiro, F.D.R. and Stallinga, P. (2020) Using an Off-the-Shelf Lock-In Detector for Admittance Spectroscopy in the Study of Plants. Agricultural Sciences, 11, 390-416. https://doi.org/10.4236/as.2020.114023

Received: November 28, 2019

Accepted: March 29, 2020

Published: April 1, 2020

Copyright (c) 2020 by author(s) and Scientific Research Publishing Inc. This work is licensed under the Creative Commons Attribution International License (CC BY 4.0).

http://creativecommons.org/licenses/by/4.0/

\begin{abstract}
We report on the development of an electrical characterization admittance spectroscopy equipment and method based on an off-the-shelf lock-in detector that is cheap and yet highly sensitive. It is concluded that a contacted constant-pressure electrode configuration is preferable. It was further determined that the temperature does not have great impact in the measured values, but relative humidity of air can be important, especially in the constant-gap electrode configuration. In-situ measurements are difficult since the coupling of the plant with the environment is of high importance. Another aspect is the cables; they are important in that they have to be terminated by their characteristic impedance (50 $\Omega$ in our case) to avoid reflections that introduce artificial attenuation and phase shifts in the signal. We introduce a fingerprint plot type to be able to distinguish between various plants and other specimens, and can actually detect the aqueous state of a plant.
\end{abstract}

\section{Keywords}

Admittance Spectroscopy, In Vivo, Non Invasive

\section{Introduction}

Irrigation in agriculture is the leading consumer of water in the world. In the end of the $21^{\text {st }}$ century, world agriculture water use reached $2290 \mathrm{~km}^{3}$ of water, $69 \%$ of the total world water usage. In southern European countries, agriculture water needs account for over $90 \%$ of overall water consumption. Population growth and economic/industrial activities are decreasing water availability everyday [1] [2]. Rising water needs make it of the essence to increase water spending efficiency through, for example, the innovation of current watering and plant assessment methods in agriculture, leading to economic gain and reduced environmental impact. A possible solution may be to electrically assess plants, estab- 
lishing patterns of normal and water-induced stress functioning and associate the internet of things concept with automation, integrating plant information and irrigation execution. This proposed system would be able to detect precisely when plants need watering as well as how much, through detection of electrical parameters from the normal functioning pattern. One fact to be taken into consideration is the enormous dielectric constant of water at room temperature (80) when compared to other materials (air 1; soil solids 2 - 5), which suggests that this system would be viable. Indeed, water has some unique characteristics [3] [4] [5], some of which we can take to our advantage, as in this case more specifically the exceptional value of the dielectric constant of water.

Many aspects of electromagnetic (EM) interaction with biological entities and their electrical properties still remain unclear and/or unknown. Generally, we can distinguish two different purposes on electrically assessing a plant: to measure long-distance signaling, usually in the form of action potentials (AP) or similar mechanisms; or to measure the electrical properties of plants, for example, by measuring several electrical parameters such as capacitance or impedance. The first approach usually tries to measure differences in the potential between two points in the plant as a way to detect cell membrane depolarization, and thus, the AP, after some kind of stimulus (such as light or cold). The latter takes a different approach in the sense that it doesn't detect AP or long-distance signaling, but instead and not only, the consequences of these. It evaluates the target site on the plant regarding electrical properties through the measurement of different electrical parameters such as capacitance, conductance, phase angle, and loss tangent which might reflect changes in structural and chemical composition. Simply putting the anatomical and physiological variations of the plant will cause changes in the plant's electrical properties, effectively transuding information, which can then be assessed through the correct equipment and technique, opening new pathways for the analysis of plant status throughout its daily and life cycle. Also, there are two ways of electrically measuring a plant, intracellular or extracellular measurements. Intracellular measurements have the downside of possible effects caused by the electrode injury which may influence results due to the sensitive nature of electrical measurement [6]. Extracellular measurement on the other hand, avoids this issue but in return, has the downside of measurements being subjected to contact resistance which, if high enough, may impede proper results, as well as being influenced by all kinds of exterior conditions such as temperature and humidity.

Regarding the study of plant long-distance signaling, it initiates its history in the $19^{\text {th }}$ century with the work of Burdon-Sanderson, Haberlandt, Pfeffer, Bose and others (reviewed in [7]). The existence of AP and long-distance signaling in plants were demonstrated and much had been advanced regarding techniques and respective optimization (reviewed in [8]). Regarding the study of electrical properties of biological tissues, it also starts in the $19^{\text {th }}$ century with the works of Du Bois-Reymond, Peltier, Hermann, D'Arsonval, Tesla and others, and it has 
also been advanced and optimized throughout the century (reviewed in [9] [10]). Noteworthy to mention are the works by Fricke, Curtis and Cole, who heavily contributed to the advancement of instrumentation and to the accurate measurement of several electrical parameters over a large frequency range [11]-[19].

More recently and relevantly, correlations between the electrical properties of a plant with its biomass, water, nutrition and stress were found. We give here a small overview:

- Greenham et al. used electrical measurements to detect changes in plant tissue derived from nutrient status [20].

- Muñoz-Huerta et al. found high and positive correlation between plant nitrogen content and plant electrical impedance in lettuce at frequencies at minimum phase angle [21].

- Tomkiewicz and Piskier assessed nutrient stressed tomato plants by electrical impedance spectroscopy [22].

- Zhang and Willison, using electrical impedance spectroscopy, showed that several electrical parameters of Brassica oleracea leaves are affected when subjected to freeze-thaw stress [6].

- Jackson and Harker used electrical impedance spectroscopy to detect the extension of damage in bruised apples [23].

- Cao et al. analyzed plant root surface area through electrical impedance spectroscopy [24].

- Jones et al. estimated plant biomass through the plant's dielectric properties [25].

- Postic and Doussan used electrical impedance spectroscopy for the estimation of plant root biomass [26].

- Van Emmerik et al. have found that, while the dielectric response of unstressed corn plants remained stable, the resonant frequency (which is dependent of the dielectric properties) of water stressed corn ear leaves was affected.

- The same researchers have also shown that water stress affects the dielectric properties of tomato leaves and that the dielectric properties of irrigated tomato plants are different than non-irrigated ones [27] [28].

- Sinha and Tabib-Azar showed that low frequency electrical capacitance and resistance of Schefflera plant leaves are affected by changes in plant hydration and light/dark cycles [29].

- He et al. developed a portable bioelectrical impedance spectroscopy system able to detect tomato plant water status [30].

- Afzal et al. estimated plant leaf moisture through measurements of the leaf's dielectric constant in five different types of plants at frequencies of $100 \mathrm{kHz}$ and $1 \mathrm{MHz}$ [31] [32].

- Jördens et al. developed an electromagnetic model to determine water content in coffee leaves through leaf permittivity at frequencies between 0.2 and 
$1.8 \mathrm{THz}[33]$.

- Burke et al. showed that Impulse Time Domain Transmission (an extension of Time Domain Reflectometry, an electromagnetic technique used for measuring the dielectric constant of soils, which is related to soil water content) is very sensitive to small changes in plant canopy water status [34].

- Chuah et al. showed that the dielectric constant of a plant leaf is a function of water content and frequency [35].

In this day and age, where economics play a vital role in research and development (R \& D) it is essential to develop technologies and cheap tools for research as we also foresee an increased proliferation of sensing in (agricultural) society in a world of the Internet of Things (IoT). In this study, we describe a novel approach to observe the response of plants to stimuli by use of a lock-in amplifier. A lock-in amplifier is basically an amplifier that is capable of obtaining a signal using a carrier wave, even when this signal has very low intensity and is present in noisy environments. Signals that are not in the same frequency as the reference signal are eliminated providing a cleaner signal. Since the objective of this study is to assess plant status throughout its daily cycle, measurements need to be of medium to long duration, that is between several days to several weeks/months, the use of electrodes glued to the leaf surface by agar (a technique commonly used) is to be avoided because the agar dries up and the electrodes disconnect from the plant. Our chosen method consisted of extracellularly (non-invasive) measuring a leaf with two parallel electrodes forming a capacitor, through a lock-in amplifier, providing a relatively cheap tool for plant extracellular electrical properties measurement.

Ideally, the ultimate goal of our research (embedded in a project of IoT) would be to be able to non-invasively determine the health of plants. Others have tried this, see for example the work of Greenham [36] [37], Borges et al. [38], Dadshani et al. [39], Dietrich et al. [40], Zhang et al. [6], Khalil et al. [41] or Martinez [42], but it remains one of the biggest challenges in agriculture. Electrical measurements in agriculture are commonly invasive by sticking needles into the measured entities [43]. Moreover, signals are often of the type of direct current (DC) action potentials (AP), with the plant being a source of energy. See for instance the article of Christman and Grill [44], the work of Fromm and Lautner [45], Mousavi et al. [46], Oyarce and Gurovich [47], Ros-Rojas et al. [48] or Yan et al. [8], or the books edited by Volkov [49] [50], while much less is known about the plant characterization by submitting them passively to signals, especially alternating current (AC) signals. The current work reports on the development of a cheap set-up for determining plant health status.

\section{Materials and Methods}

\section{Electronic setup}

The experimental setup is designed to measure the impedance $(Z)$ or admittance $(Y=1 / Z)$ of a plant leaf in vivo. This is done by placing the leaf between 
two electrodes, see Figure 1. Maxwell's equations tell us how much capacitance ( $C$ ) and resistance $(R)$ a bar of material has. A block of material with area $A$ and thickness $d$ has a capacitance given by

$$
C=\frac{\varepsilon_{\mathrm{r}} \varepsilon_{0} A}{d},
$$

with $\varepsilon$ the permittivity of the material equal to the dielectric constant ( $\varepsilon_{\mathrm{r}}$, often called $K$ in literature) times the permittivity of vacuum $\left(8.85 \times 10^{-12} \mathrm{~F} / \mathrm{m}\right)$, $\varepsilon=\varepsilon_{\mathrm{r}} \varepsilon_{0}$. Likewise, the resistance of this bar is given by

$$
R=\frac{\rho d}{A}
$$

with $\rho$ the resistivity of the material. The complex admittance of this bar is then the parallel sum of the conductance of the resistor $(G=1 / R)$ and susceptance of the capacitor $(B=\omega C)$,

$$
Y=G+j B=\frac{1}{R}+j \omega C,
$$

with $j^{2}=-1$ and $\omega$ the frequency of oscillation. If we apply a sinusoidal voltage, $V(t)=V_{0} \sin (\omega t)$ (in phasor analysis: $V(t)=V_{0} \mathrm{e}^{j \omega t}$ ), a current will flow through the device given by Ohm's law, $I(t)=V(t) / Z=V(t) Y$, which, generally speaking, has a real and imaginary part, the former being in phase with the applied voltage and the latter being 90 degrees ahead of it. The above equation shows that the real part is associated with the parallel resistance and the imaginary part to the parallel capacitance,

$$
\begin{gathered}
I(t)=I_{0} \sin (\omega t)+I_{90} \cos (\omega t), \\
I_{0}=V_{0} / R, \\
I_{90}=\omega V_{0} C .
\end{gathered}
$$

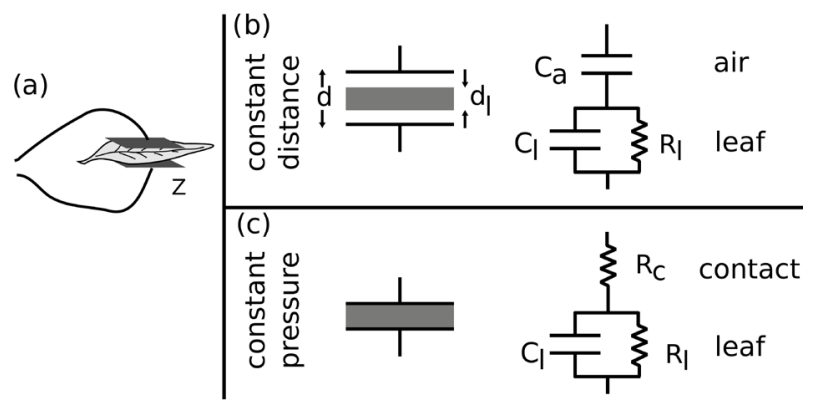

Figure 1. Basic scheme. (a) Measurement of a leaf between electrodes the total having an impedance $Z$ that will be measured. (b) Schematic representation of a in a contactless constant-gap configuration with electronic equivalent circuit of the impedance of the electrodes that can be seen as composed of capacitance and resistance of the leaf in series with capacitance and (infinite, hence not shown) resistance of the air, the part of the electrodes that is not filled with plant, c) Schematic representation in a contacted constant-pressure configuration with electronic equivalent circuit of the impedance of the electrodes that can be seen as composed of capacitance and resistance of the leaf in series with contact resistance. 
The latter equation we could also have arrived at if we had started with the definition of capacitance as the capacity to store charge, $C \equiv Q / V$, or $Q=V C$, and where current is charge flowing, the current of a (constant) capacitor submitted to a sine wave voltage is then $I_{C}=C \mathrm{~d} V / \mathrm{d} t=\omega V_{0} C \cos (\omega t)$. The current through a resistor is directly equal to the ratio of voltage and resistance, as Ohm's law tell us and thus results in a sine wave current when a sine wave voltage is applied, $I_{R}=\left(V_{0} / R\right) \sin (\omega t)$ (This approach has the advantage of not including non-physical imaginary numbers).

Determining resistance and capacitance of a sample such as a leaf between electrodes then consists of measuring the magnitudes of currents in-phase and 90-degrees-out-of-phase with the applied voltage. Admittance spectroscopy (or impedance spectroscopy) then is nothing more than determining these parameters as a function of frequency $\omega$ and, additionally, these parameters can also be measured as a function of time, in so called "transients". We'll measure parallel capacitance and resistance as a function of time and frequency, through the continuous measurement of in-phase and out-of-phase current magnitudes with a constant-amplitude sine voltage applied:

$$
\begin{gathered}
R_{\mathrm{p}}(\omega, t)=\frac{V_{0}}{I_{0}(\omega, t)}, \\
C_{\mathrm{p}}(\omega, t)=\frac{I_{90}(\omega, t)}{\omega V_{0}} .
\end{gathered}
$$

We call these parameters $R_{\mathrm{p}}$ and $C_{\mathrm{p}}$ the as-measured resistance and capacitance, respectively. (The subscript "p" denotes "parallel"; most commercial equipment use this convention of representing the total admittance of a device by a resistor in parallel with a capacitor). This is the main focus of this paper. Admittance spectroscopy basically entails applying a sine wave voltage and measuring the in-phase and out-of-phase components of the current from which capacitance and resistance can be derived.

Other parameters (combinations of the two above) might also be useful. For instance, the product of capacitance and resistance is a parameter with unit time that does not depend on the device dimensions, as it depends only on the material properties: $\tau=R C=\varepsilon_{\mathrm{r}} \varepsilon_{0} \rho$, and this time can represent an important process going on in the measured object, such as charge-trapping relaxation times. Another often-used parameter in spectroscopy is the phase angle, or loss tangent. Loss defined as $L=1 / \omega R$ (unit: farad), the phase angle is the angle in an impedance polar plot, $\delta \equiv \angle Z$, the tangent of which is called the loss tangent,

$$
\tan \delta \equiv \frac{j Z_{\mathrm{C}}}{Z_{\mathrm{R}}}=\frac{1 / \omega C}{R}=\frac{1}{\omega R C}=\frac{L}{C},
$$

which can give meaningful information, as will be shown. The phase angle can also be defined as the angle in an admittance plot, $\theta \equiv \angle Y$ and given the fact 
that $Y=1 / Z$ the phase angle is $\theta=90^{\circ}-\delta$. The data can also be shown in a so-called Cole-Cole plot of loss vs. capacitance, which for a single-time-constant ( $\tau=R C$ ) systems is a semi circle with a top (maximum loss) reached at a frequency equal to $\omega=1 / \tau$.

These are the standard characterization techniques we have at our disposition, which we will also use in this work.

\section{The lock-in detector}

To measure these parameters ( $Z$ in general, see Figure 2 ) we use a standard lock-in detector that has an oscillator output (labeled $V_{\text {ref }}$ ) and a two-channel, phase-sensitive, amplifier, detecting the in-phase and out-of-phase components of a voltage input (labeled $V_{\text {in }}$ ), the in-phase called $\mathrm{x}$ and out-of-phase $\mathrm{y}$. The current through $Z$ is basically sampled by the shunt resistor $R_{\mathrm{s}}, I=V_{\text {in }} / R_{\mathrm{s}}$ and from there $Z$ is calculated, which is given by the approximation $Z=V_{\text {ref }} / I$, which is valid if $\left|R_{\mathrm{s}}\right| \ll|Z|$, so that $Z$ feels the entire reference voltage, with one leg of $Z$ effectively as good as grounded.

A problem arises that the oscillator output voltage source has an output resistance given by $R_{\mathrm{o}}$ (typically $50-100 \Omega$ ), and the input impedance of the lock-in detector is given by a resistor $R_{\text {in }}$ typically $10 \mathrm{M} \Omega$ in parallel with a capacitor $C_{\mathrm{in}}$, typically $20 \mathrm{pF}$ ). Figure 2 gives the full equivalent circuit used in measuring. The simplified circuit on the right of the figure shows that the oscillator reference voltage enters a voltage divider that results in an input voltage for the lock-in detector equal to

$$
V_{\text {in }}=V_{\text {ref }} \frac{Z_{\text {eq }}}{Z_{\text {eq }}+\left(Z+R_{\mathrm{o}}\right)} \text {, }
$$

with

$$
\begin{aligned}
Z_{\text {eq }} & =\left(R_{\mathrm{s}} / / R_{\text {in }} / / C_{\text {in }}\right) \\
& =\frac{R_{\mathrm{s}} R_{\mathrm{in}}}{\left(R_{\mathrm{s}}+R_{\mathrm{in}}\right)+j \omega R_{\mathrm{s}} R_{\mathrm{in}} C_{\mathrm{in}}} \\
& =\beta_{\mathrm{r}}+j \beta_{\mathrm{i}},
\end{aligned}
$$

in which

(a)

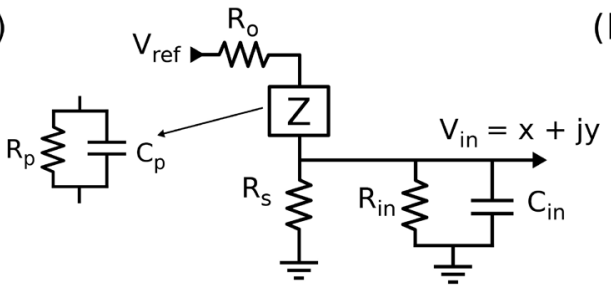

(b)

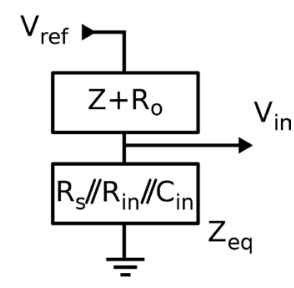

Figure 2. Electronic equivalent circuit. (a) Experimental setup. The measured sample $\mathrm{Z}$ is imagined to be composed of a resistor $R_{\mathrm{p}}$ in parallel with a capacitor $C_{\mathrm{p}} . R_{\mathrm{o}}$ is the output resistance of the oscillator voltage source $V_{\text {ref. }}$ The input impedance of the lock-in detector is a resistance $R_{\text {in }}$ typically in the order of 10 to 100 megaohms, in parallel with a capacitor $C_{\mathrm{in}}$ in the order of tens of picofarads. The voltage-divider equivalent circuit is given in (b). 


$$
\begin{aligned}
& \beta_{\mathrm{r}}=\frac{R_{\mathrm{s}} R_{\text {in }}\left(R_{\mathrm{s}}+R_{\text {in }}\right)}{\left(R_{\mathrm{s}}+R_{\text {in }}\right)^{2}+\left(\omega R_{\mathrm{s}} R_{\text {in }} C_{\text {in }}\right)^{2}}, \\
& \beta_{\mathrm{i}}=-\frac{\omega\left(R_{\mathrm{s}} R_{\text {in }}\right)^{2} C_{\text {in }}}{\left(R_{\mathrm{s}}+R_{\text {in }}\right)^{2}+\left(\omega R_{\mathrm{s}} R_{\text {in }} C_{\text {in }}\right)^{2}} .
\end{aligned}
$$

Equation (7) rewritten:

$$
\begin{aligned}
Z & =Z_{\text {eq }} \frac{V_{\text {ref }}}{V_{\text {in }}}-\left(Z_{\text {eq }}+R_{\mathrm{o}}\right) \\
& =\left(\beta_{\mathrm{r}}+j \beta_{\mathrm{i}}\right) \frac{V_{\text {ref }}}{V_{\text {in }}}-\left(\beta_{\mathrm{r}}+j \beta_{\mathrm{i}}\right)-R_{\mathrm{o}}
\end{aligned}
$$

The input voltage is composed of an in-phase signal $(x)$ and 90-degrees-out-of phase component (y). Substituting $V_{\text {in }}=x+j y$ gives

$$
\mathrm{Z}=\alpha_{\mathrm{r}}+j \alpha_{\mathrm{i}}
$$

with

$$
\begin{aligned}
& \alpha_{\mathrm{r}}=\frac{V_{\text {ref }}}{x^{2}+y^{2}}\left(\beta_{\mathrm{r}} x+\beta_{\mathrm{i}} y\right)-\beta_{\mathrm{r}}-R_{\mathrm{o}}, \\
& \alpha_{\mathrm{i}}=\frac{V_{\text {ref }}}{x^{2}+y^{2}}\left(\beta_{\mathrm{i}} x-\beta_{\mathrm{r}} y\right)-\beta_{\mathrm{i}} .
\end{aligned}
$$

The admittance $Y$ is the reciprocal of the impedance $Z$ :

$$
Y \equiv \frac{1}{Z}=\frac{1}{\alpha_{\mathrm{r}}+j \alpha_{\mathrm{i}}}=\frac{\alpha_{\mathrm{r}}}{\alpha_{\mathrm{r}}^{2}+\alpha_{\mathrm{i}}^{2}}-j \frac{\alpha_{i}}{\alpha_{\mathrm{r}}^{2}+\alpha_{\mathrm{i}}^{2}}
$$

If we assume the impedance box $Z$ (or $Y$ ) is composed of a resistor in parallel with a capacitor, as shown in Figure 2, which has a total admittance of

$$
Y=\frac{1}{R_{\mathrm{p}}}+j \omega C_{\mathrm{p}},
$$

we measure resistance and capacitance values equal to, resp.

$$
\begin{aligned}
& R_{\mathrm{p}}=\frac{\alpha_{\mathrm{r}}^{2}+\alpha_{\mathrm{i}}^{2}}{\alpha_{\mathrm{r}}}, \\
& C_{\mathrm{p}}=-\frac{\alpha_{\mathrm{i}}}{\omega\left(\alpha_{\mathrm{r}}^{2}+\alpha_{\mathrm{i}}^{2}\right)} .
\end{aligned}
$$

In an ideal measurement setup, the lock-in detector reference voltage source has zero output resistance, the input has infinite input resistance and no stray capacitance. Moreover, we chose the measuring shunt resistance to be tiny, so, in the limit,

$$
R_{\mathrm{o}}=0, R_{\mathrm{in}}=\infty, C_{\mathrm{in}}=0,\left|R_{\mathrm{s}}\right| \ll|Z| \text {, }
$$

and this implies

$$
\begin{aligned}
& \beta_{\mathrm{r}}=R_{\mathrm{s}}, \beta_{\mathrm{i}}=0, \\
& \alpha_{\mathrm{r}}=\frac{V_{\text {ref }} R_{\mathrm{s}} x}{x^{2}+y^{2}}, \alpha_{\mathrm{i}}=-\frac{V_{\mathrm{ref}} R_{\mathrm{s}} y}{x^{2}+y^{2}} .
\end{aligned}
$$


This results in the simplified measured values of

$$
\begin{aligned}
& R_{\mathrm{p}}=\frac{V_{\mathrm{ref}} R_{\mathrm{s}}}{x}, \\
& C_{\mathrm{p}}=\frac{y}{\omega V_{\text {ref }} R_{\mathrm{s}}},
\end{aligned}
$$

which is equal to the earlier found values (Equation (5)) if we realize that the voltage in $x$ is equal to $R_{\mathrm{s}} I_{0}$ and in $y$ equal to $R_{\mathrm{s}} I_{90}$ and $V_{\text {ref }}=V_{0}$. Note that, under normal conditions, the correction factor between the simplified equation and the full equation is minimal, but care has to be taken to not let the shunt resistance $R_{\mathrm{s}}$ get too high, or non-linear effects and phase shifts (conductance appearing as capacitance, and vice versa) may occur. Also, this resistance should not be too low, or the signal will get too noisy. Input signals for both $x$ and $y$ bigger than $0.1 \%$ to $1 \%$ of the reference voltage amplitude should be avoided. In some lock-in detectors directly current can be measured and this simplifies the conversion. However, not all lock-in detectors have this feature and we limited ourselves to using voltage input. We conclude that a standard lock-in detector can be used for admittance spectroscopy if care is taken.

A final note is that the value of $R_{\mathrm{s}}$ can be dependent on temperature and even on humidity, and this directly influences the values of as-measured resistance and capacitance as the above equation shows. However, these effects we deem to be of minor importance, of the order of $0.1 \% /{ }^{\circ} \mathrm{C}$.

\section{The Cables}

The cables used to connect the impedance $Z$ to the lock-in detector are preferentially of coaxial type, a metal core inside another cylindrical shield, separated by an insulator, for instance polytetrafluoroethylene (PTFE). This because it will shield any stray capacitance of the wire with the surroundings from interfering and reduces the noise level significantly. Transmission-line analysis shows us that such a cable has capacitance and inductance itself (both per meter cable length), but the total impedance of the cable is ohmic and this value depends on the diameters of the cylinders and the dielectric properties of the insulator [51]. Typically a cable has a characteristic impedance of $Z_{0}=50 \Omega$ resistance. The importance of this resistance value is that at the end of the cable the signal is reflected back with a factor

$$
r=\frac{Z_{\mathrm{L}}-Z_{0}}{Z_{\mathrm{L}}+Z_{0}},
$$

with $Z_{\mathrm{L}}$ the load impedance, in our case basically $R_{\mathrm{s}}$. The signal coming from the sample arrives at the lock-in detector and is reflected back to the sample with a factor $r$. At the sample $Z$ the same thing happens, the signal is reflected (with a different factor $r^{\prime}$ ). This doubly-reflected signal arrives with a time delay relative to the first signal at the lock-in detector, $\Delta t=2 \Lambda / v$ (with $\Lambda$ the cable length and $v$ the speed of light in PTFE, about $2 \times 10^{8} \mathrm{~m} / \mathrm{s}$ ), see Figure $3(\mathrm{a})$. And reflected again, coming back as a quadruply-reflected signal, etc. For high load re- 
sistances and close-to-unity reflection coefficients $r$, the number of reflections can be enormous. Interference effects between all these waves can cause distortion of the signal. To give an example, imagine we have a shunt resistance equal to $10 \mathrm{k} \Omega$ and an infinite sample impedance, $Z=\infty$. The reflection at the sample is unity and at the lock-in detector equal to

$$
r \approx 1-2 \times 50 \Omega / 10 \mathrm{k} \Omega=0.99 \text {. }
$$

For a reference frequency $\omega$ we can calculate the total interference function arriving at the lock-in detector as a sum of $2 n$-fold-reflected waves,

$$
V_{\text {in }}=\sum_{i=0}^{\infty} r^{i} \sin \left[\omega\left(t-\frac{2 i \Lambda}{v}\right)\right],
$$

It will start seriously interfering with the signal when the amplitude $r^{i}$ of the component at a phase shift of $180^{\circ}$ is still significant, at an $i$ in the neighborhood where $\omega 2 i \Lambda / v=\pi$. In that case the interference function is a fully randomized (read: zero magnitude) wave at the input.

Analyzing the above equation, when these effects occur and hinder our measurements we can thus do either one of the following:

- Reduce the cable length, $\Lambda$, if practical.

- Reduce the shunt resistance $R_{\mathrm{s}}$. At $50 \Omega$ these problems are completely gone $(r=0)$. This we call impedance matching. However, it also reduces the amplitude at the entrance terminal of the lock-in detector and the final signal will thus be more noisy.

- Reduce the reference frequency $\omega$, but this will also reduce the range of usable frequencies which eliminates the usefulness of impedance spectroscopy.

(a)

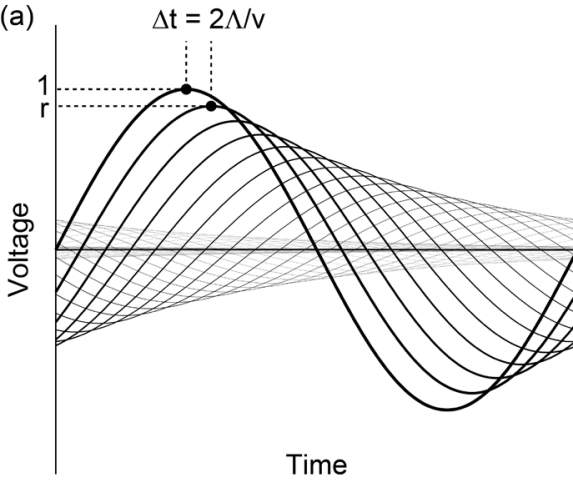

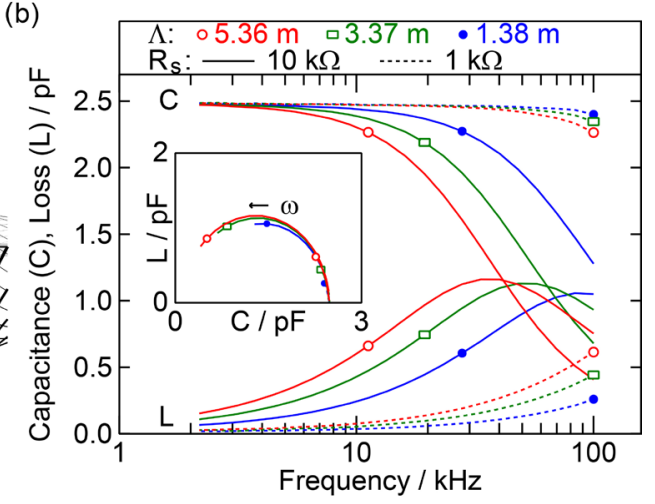

Figure 3. Effects of the cables. (a) Multiple reflections in a non-impedance-matched cable

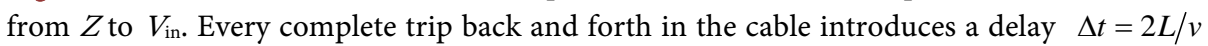
and an attenuation $r$. If the attenuation is not fast enough compared to the phase change, destructive interference can occur. (b) Experimental effect of cable length on the spectrum of a system of empty electrodes (capacitance, top plots and loss, bottom plots). Shunt resistance $R_{\mathrm{s}}$ $=10 \mathrm{k} \Omega$. Cable lengths: $1.38 \mathrm{~m}$ (rightmost plot), $3.37 \mathrm{~m}$ (middle plot) and $5.36 \mathrm{~m}$ (leftmost plot). The dashed lines repeat the experiment for a shunt resistance of $R_{\mathrm{s}}=1 \mathrm{k} \Omega$. Note that the cable length from $V_{\text {ref }}$ to $Z$ had no effect since the output of the lock-in detector was phase matched at $50 \Omega$ and no reflections took place there. 
Figure 3(b) shows an example of the effect of cable length (from $Z$ to $V_{\text {in }}$ ) on the spectrum. A cut-off frequency is clearly discerned that moves to lower values when increasing the cable length. Changing the shunt resistance to lower values also changes it.

The other cable, from $V_{\text {ref }}$ to $Z$ does not suffer from this effect if the output resistance of the lock-in detector is impedance matched at $50 \Omega$ and no reflections take place there.

\section{Biological applications}

For biological applications such as measuring leaves performed here, the first problem arises that, generally speaking, the electrodes do not make intimate contact with the leaf and we are not only measuring the leaf. There are then two configurations of measuring possible, as depicted in Figure 1. In the first configuration, we take a constant-distance electrode ( $d$ fixed). This implies that the electrodes are partly filled with air and this has high resistance $\left(R_{\mathrm{a}}\right)$, which we thus take as infinite. (In what follows, a subscript "a" signifies "air", while "l" and "c" mean "leaf" and "contact" respectively). The total as-measured capacitance and resistance defined in Equation (5) can be found as

$$
\begin{aligned}
& R_{\mathrm{p}}=\frac{1+\omega^{2} R_{1}^{2}\left(C_{\mathrm{a}}+C_{\mathrm{l}}\right)^{2}}{\omega^{2} R_{\mathrm{l}} C_{\mathrm{a}}^{2}}, \\
& C_{\mathrm{p}}=\frac{C_{\mathrm{a}}+\omega^{2} R_{1}^{2} C_{\mathrm{a}} C_{1}\left(C_{\mathrm{a}}+C_{1}\right)}{1+\omega^{2} R_{1}^{2}\left(C_{\mathrm{a}}+C_{\mathrm{l}}\right)^{2}} .
\end{aligned}
$$

Note that even if all parameters are independent of frequency, the as-measured capacitance and resistance $\left(C_{\mathrm{p}}\right.$ and $\left.R_{\mathrm{p}}\right)$ do depend on frequency. For low frequencies, the above equations approximate to

$$
\begin{aligned}
& R_{p}=\infty, \\
& C_{\mathrm{p}}=C_{\mathrm{a}}=\frac{\varepsilon_{\mathrm{a}} \varepsilon_{0} A}{d-d_{1}} .
\end{aligned}
$$

and we measure effectively the thickness of the leaf (or the permittivity of air, which is rather featureless). While for high frequencies this tends to

$$
\begin{aligned}
& R_{\mathrm{p}}=R_{l} \frac{\left(C_{\mathrm{a}}+C_{\mathrm{l}}\right)^{2}}{C_{\mathrm{a}}^{2}}, \\
& C_{\mathrm{p}}=C_{\mathrm{a}} \oplus C_{\mathrm{l}} .
\end{aligned}
$$

(The $\oplus$ symbol signifying the series-sum of two capacitors in series).

To get an idea, in our case the area of the electrodes was about $A=4 \mathrm{~cm}^{2}$, with a distance of $d=1.8 \mathrm{~mm}$, a leaf thickness of $d_{l}=0.2 \mathrm{~mm}$ and a dielectric constant of air equal to 1 and of water equal to $80, \rho_{\mathrm{a}}=2 \times 10^{14} \Omega \cdot \mathrm{m}$,

$\rho_{1}=200 \Omega \cdot \mathrm{m}$, we estimate the resistance of air to be $R_{a}=800 \mathrm{~T} \Omega$ (justifying our assumption it is infinite), $C_{a}=2.2 \mathrm{pF}, R_{l}=6 \mathrm{k} \Omega$, and $C_{l}=1.4 \mathrm{nF}$. And the cut-off frequency (where the loss tangent peaks) is $9.2 \mathrm{MHz}$.

If on the other hand the electrodes make intimate contact with the leaf, effec- 
tively we have the situation as in Figure 1(d) and Figure 1(e). The distance between the electrodes is now not constant, but in the ideal case equal to the leaf thickness. A residual contact resistance might exist, making designing a proper way to contact the leaf essential if the objective is to measure the plant.

The as-measured resistance and capacitance become

$$
\begin{aligned}
& R_{\mathrm{p}}=\frac{\left(R_{1}+R_{\mathrm{c}}\right)^{2}+\omega^{2} R_{1}^{2} R_{\mathrm{c}}^{2} C_{1}^{2}}{\left(R_{1}+R_{\mathrm{c}}\right)+\omega^{2} R_{1}^{2} R_{\mathrm{c}} C_{1}^{2}}, \\
& C_{\mathrm{p}}=\frac{R_{1}^{2} C_{l}}{\left(R_{1}+R_{\mathrm{c}}\right)^{2}+\omega^{2} R_{1}^{2} R_{\mathrm{c}}^{2} C_{l}^{2}} .
\end{aligned}
$$

which again depend on the frequency even if the parameters don't. For lousy contact $\left(R_{\mathrm{c}} \rightarrow \infty\right)$, we measure just the contact resistance $\left(R_{\mathrm{p}}=R_{\mathrm{c}}, C_{\mathrm{p}}=0\right)$, nothing more. On the other hand, good contact can be ensured by using Agar as contacting electrolyte, much similar to the gel that is used when taking electrocardiograms. It basically avoids any contacting problems such as irregularity of surface and micro air bubbles. For perfect contact, $R_{\mathrm{c}}$ is zero and we measure the capacitance and resistance of the leaf, which might, however, effectively be just measuring the thickness, where the intricacies of plant state could more be in the dielectric constant $\varepsilon_{1}(\omega)$,

$$
\begin{aligned}
& R_{\mathrm{p}}=R_{\mathrm{l}}=\frac{\rho_{\mathrm{l}} d_{1}}{A}, \\
& C_{\mathrm{p}}=C_{\mathrm{l}}=\frac{\varepsilon_{1} \varepsilon_{0} A}{d_{1}} .
\end{aligned}
$$

The RC-time-constant measurement techniques of phase angle tangent (Equation (6)) do reveal the internal plant state

$$
\tan \delta=\frac{1}{\omega \varepsilon_{0} \rho_{1}(\omega) \varepsilon_{1}(\omega)} .
$$

We conclude that the best way to measure plants is to use contacted electrodes and ensure good contacts. The information of the plant state is then best found in the phase angle, unless one is interested in the leaf thickness (water content). The best way to ensure good contacts is with agar, gluing the electrodes to the surface of the leaf. However, agar dries up and thus this is only possible for measurement times that are faster than the typical drying time, in the order of hours. In the measurements presented here were refrained from using agar. Our advice to the reader is to determine for each experiment what is the best way to contact.

\section{The electrodes}

A problem with the measurement of admittance, a problem that is not limited to our measurement set-up but rather common for electrical measurements, is that it is very difficult to measure the subject under study independent of the rest of the world. As an example, a plant is rather conductive and if we place a leaf of the plant between the electrodes and the leaf is still connected to the plant that is 
in turn rooted in the ground, it is unavoidable that we will also measure the plant and even the environment in which the plant is placed. In the ultimate case the measurements actually may be just measuring the environmental changes and have nothing to do with the plant, nor the leaf that is supposedly being measured. An example is shown in Figure 4(a). A plant is measured in-vivo by placing a leaf between the electrodes. The nerves of the leaf are conductive and thus well electrically connected to the rest of the plant and to the ground. The ground is in a vase that is on a metal shelf of a closet. The equivalent circuit is shown in Figure 4(b). Basically, the system measures the conductivity of the earth since that has most effect on the measured value. To understand it, imagine we give water to the plant and reduce the resistance $R_{\text {earth }}$ to zero, basically the nerves of the leaf are in short circuit with the closet. The effect is that the electric field induced by the reference voltage $V_{\text {ref }}$ on the top electrode does not reach the bottom electrode anymore and thus no current is induced in the input shunt resistance $R_{\mathrm{s}}$ at the lock-in detector and $V_{\text {in }}$ will be zero. Even while the leaf itself has capacitance and conductance, none will be measured. Thus, the system is highly sensitive to changes of soil humidity, something that is probably not desirable.

A solution we propose is to wrap the vase in aluminum foil and connect it electrically well to the stem of the trunk of the plant. In this way there will be always good contact between plant and the rest of the world, so that giving water to the soil in the vase does not modulate it. It is, of course, a cheap man's solution, but it allows for observing variations in plant state. An alternative is to isolate the plant very well, but this is more difficult and impractical than it may seem.

\section{The effect of the environment}

After having established the electronic set up and determined what is the best way to measure it, some details about the effect of the environmental conditions on the measurements, such as temperature and (relative) humidity, remain to be described. This in order to be able to distinguish them from the real effects of the biological entities measured. Let's start with the effect of temperature.

When we look at the capacitance given in Equation (1) we see that it depends on the temperature through the dependence of $\varepsilon_{\mathrm{r}}, A$ and $d$, and the total dependence

(a)

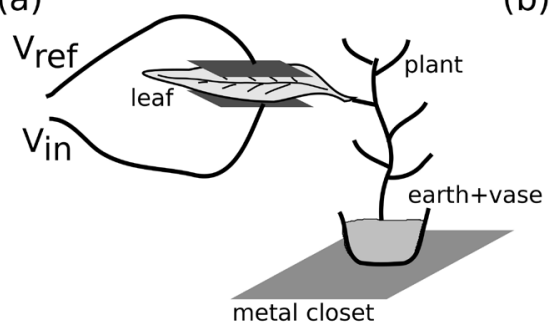

(b)

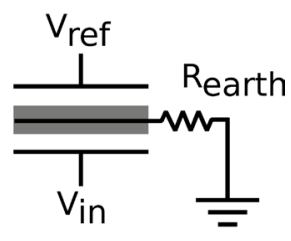

Figure 4. Connection to the environment. (a) Situation of a plant leaf measured in-vivo. The leaf is still connected to a plant and the plant is in a vase. The leaf (veins) are conductive and this basically grounds a plate which shields the bottom electrode from electric fields of the top electrode. Little signal will reach $V_{\text {in }}$. 
can be expressed in relative terms of these dependencies which are all well known,

$$
\frac{\mathrm{d} C}{\mathrm{~d} T}=\left[\frac{1}{\varepsilon_{\mathrm{r}}} \frac{\mathrm{d} \varepsilon_{\mathrm{r}}}{\mathrm{d} T}+\frac{1}{A} \frac{\mathrm{d} A}{\mathrm{~d} T}-\frac{1}{d} \frac{\mathrm{d} d}{\mathrm{~d} T}\right] \times C \text {, or } \frac{\mathrm{d} C / \mathrm{d} T}{C}=\alpha_{\varepsilon}+\alpha_{\mathrm{A}}-\alpha_{\mathrm{d}},
$$

defining the three temperature dependences $\alpha$. As an example, for a configuration of aluminum electrodes glued on acrylic plates sandwiching air (Figure 1 (b), without the leaf), $\varepsilon_{\mathrm{r}}$ is the one of air (very close to 1), with a temperature dependence of $\alpha_{\varepsilon}=-1.94 \times 10^{-6} / \mathrm{K}$ (Table 2 of Ref. [52]), $A$ is governed by the linear thermal expansion coefficient of acrylic which has to be multiplied by 2 to give the areal expansion coefficient, thus $\alpha_{\mathrm{A}}=140 \times 10^{-6} / \mathrm{K}$ [53], and $d$ is governed by the linear thermal expansion coefficient of the steel screws used for separating the acrylic plates, $\alpha_{\mathrm{d}}=12 \times 10^{-6} / \mathrm{K}$ [54]. We see that the thermal expansion of the acrylic plates is dominating the temperature dependence. In case we use a variable-distance electrode configuration of electrodes contacting the leaf, as in Figure 1(d), the inter-electrode distance $d$ is governed by the thermal expansion coefficient of the plant, which is mostly water $\left(\alpha_{\mathrm{d}}=60 \times 10^{-6} / \mathrm{K}\right)$. This is still smaller than the thermal expansion effect of the acrylic plates. Similarly, $\varepsilon_{\mathrm{r}}$ is then the one of the watery plant (the dielectric constant of foliage is in the range 30-70 [Figure 2 of Ref. [55]], where water has 80) and we estimate $\alpha_{\varepsilon}=-4500 \times 10^{-6} / \mathrm{K}[56]$ ), which now fully dominates. In the experimental results section we will refer to these theoretical calculations.

Likewise, for the resistance (Equation (2)),

$$
\frac{\mathrm{d} R}{\mathrm{~d} T}=\left[\frac{1}{\rho} \frac{\mathrm{d} \rho}{\mathrm{d} T}+\frac{1}{\mathrm{~d}} \frac{\mathrm{d} d}{\mathrm{~d} T}-\frac{1}{A} \frac{\mathrm{d} A}{\mathrm{~d} T}\right] \times R, \text { or } \frac{\mathrm{d} R / \mathrm{d} T}{R}=\alpha_{\rho}+\alpha_{\mathrm{d}}-\alpha_{\mathrm{A}},
$$

where the first term is difficult to ascertain for many materials (including air, which is further complicated by the important humidity effects). Resistance and loss being linearly linked by frequency, the same equation above for relative effects of temperature can be applied to loss.

$$
\frac{\mathrm{d} L / \mathrm{d} T}{L}=\alpha_{\rho}+\alpha_{\mathrm{d}}-\alpha_{\mathrm{A}} .
$$

Especially in case of a constant-electrode distance configuration (partly) filled with air, the effect of (relative) humidity $(\mathrm{RH})$ of the air $(\phi)$ can also be large (for a configuration with contacts to the plants the RH of air should have no effect). The other two terms are the same as above. In this case the dependence can be given as

$$
\begin{aligned}
& \beta_{\varepsilon} \equiv \frac{\mathrm{d} C / \mathrm{d} \phi}{C}=\left[\frac{1}{\varepsilon_{\mathrm{r}}} \frac{\mathrm{d} \varepsilon_{\mathrm{r}}}{\mathrm{d} \phi}\right], \\
& \beta_{\rho} \equiv \frac{\mathrm{d} R / \mathrm{d} \phi}{R}=\left[\frac{1}{\rho} \frac{\mathrm{d} \rho}{\mathrm{d} \phi}\right],
\end{aligned}
$$

with $\beta_{\varepsilon}=2.03 \times 10^{-6} / \%$ (Table 3 of Ref. [52]) and $\beta_{\rho}$ not well determined but from Pawar et al. a rough value of $0.2 / \%$ can be derived from their air-above seawater experiments [57]. The difficulty of measuring this latter parameter is 
due to the fact that air, even if it is moist, is highly insulating. We anyway do not expect the resistivity of air to play a role in the experiments. If conductivity is observed, it is rather due to leakage currents, or because the biological specimen is conducting.

\section{Practical set up}

For the experiments two equal dual-channel lock-in amplifiers of Stanford Research Systems were used (model SR830 DSP). In normal operation of measuring transients, one measured the biological specimen being studied, while the other measured the reference system, for instance a piece of paper or a plant imitation. In these cases both lock-in amplifiers were operating with a sine wave amplitude of $0.1 \mathrm{~V}$ but at slightly different frequencies to avoid interference. Typically $10.00 \mathrm{kHz}$ and $10.02 \mathrm{kHz}$, where the difference frequency of $20 \mathrm{~Hz}$ falls outside the low-pass filter range (the reciprocal RC time constant; typically $10 \mathrm{~s}$ ) of the lock-in detectors, while still ensuring similarity for the experiments. Both lock-in detectors were controlled through RS232 by computers running custom-made acquisition software that typically averaged 160 readings to result in ca. 1 data point every 10 seconds. Analysis of the data was done off-line on other computers by a range of commercial and custom-made softwares. The final plots were made by the graphic Encapsulated Postscript (EPS) toolbox XPSTool.

A steel closet was adapted by installing ventilation fans and white LED lighting so as to act both as a cultivation and measurement site (acting as a Faraday cage) and grounded to a power socket's ground. A temperature/humidity Arduino sensor (DHT22) was connected through RS232 to the same computer receiving the lock-in amplifier data. Electrodes used for measuring were of aluminum, circle shaped and with ca. $2 \mathrm{~cm}^{2}$ of area. A common chemistry clamp stand and clamp set with attached electrodes were used as apparatus as it provides malleability and versatility on positioning electrodes while maintaining the original position and structural integrity of the plant leaf. The distance between electrodes was adjustable and in the case of contacted measurements fixed to the point of ensuring good contact but not enough pressure to disturb significantly the leaf's normal functioning.

Chosen plants for measuring were Stevia rebaudiana and Mentha spicata mainly due to accessibility. Plants were watered once a week with $150 \mathrm{~mL}$ of regular tap water and once every three months with a nutrients solution diluted in tap water containing supplements of amino acids, nitrogen, phosphorus, potassium and others (Nutramin-P, Lot \#042016, Sybiol). A neonicotinoid insecticide was used when necessary (Polysect Ultra Pronto, Ref. Liscampo 6140, Scotts France SAS). The plants being measured were subjected to different schemes of light/dark hours according to different experiments, under white LED lighting, inside a closed, ventilated metal cage. The rest of them were subjected to natural lighting conditions. Figure 5 shows a photograph of the set up with a Mentha spicata plant. 


\section{Results and Discussion}

In order to characterize the lock-in amplifier, we first proceeded to do a noise analysis. We measured the capacitance of a capacitor of $100 \mathrm{pF}$ throughout time, varying frequency, ceteris paribus. The noise was determined as the standard deviation of the signal of 10-minute measurements. We could observe what was theoretically predicted: the noise is lower when increasing frequency, falling off linearly with frequency (so-called $1 / \mathrm{f}$ noise) until hitting the digitalization noise level of the amplifier (the difference between two possible digital values of the amplifier), and at frequencies of $50 \mathrm{~Hz}$ and its overtones the noise is substantially higher. See Figure 6 . The conclusion we can draw is that measuring at higher frequencies is advisable (but we have to be aware that important information might be lost) and that in any case the power line frequency and its overtones have to be avoided.

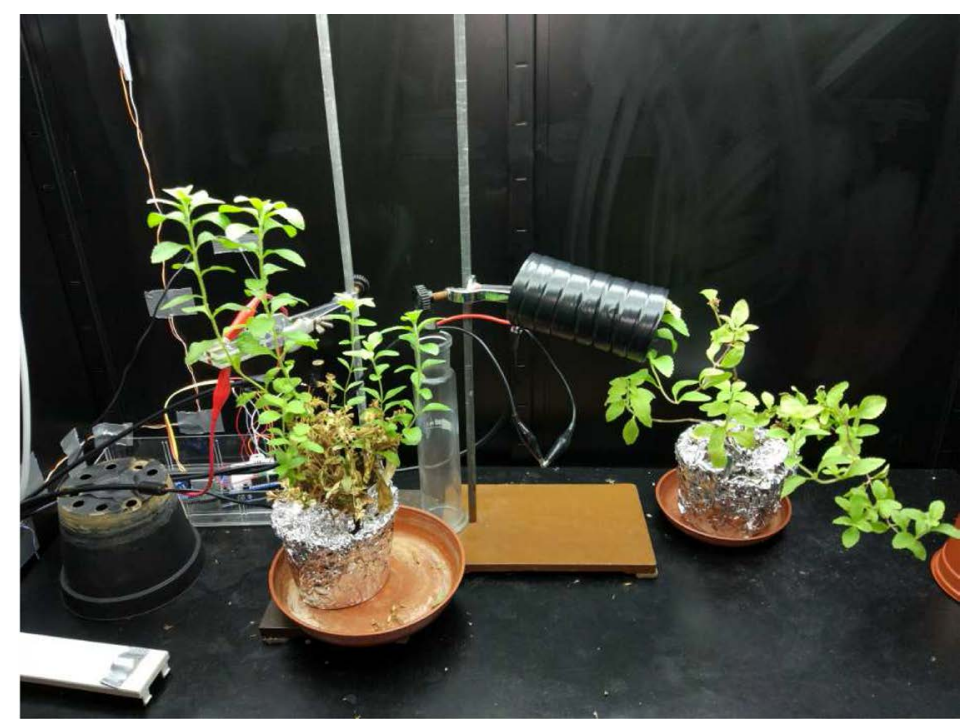

Figure 5. Photograph of the experimental set up with a Mentha Spicata plant.

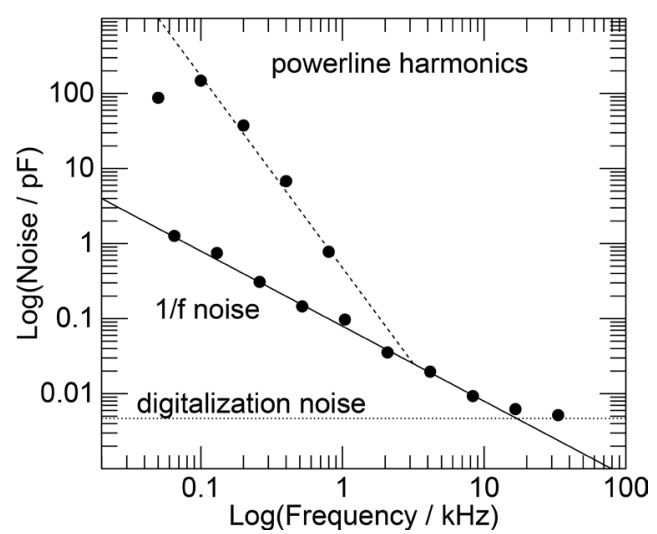

Figure 6. Noise level as a function of frequency. It shows the source for the noise is of $1 / \mathrm{f}$ character with higher noise at harmonics of the power line frequency $(50 \mathrm{~Hz})$. At higher frequencies the signal is basically noise-less and only limited by the digitalization noise of the amplifier (the difference between two possible signal levels). 
The next step was taking a measurement of spectra of the loss-tangent of a leaf of a Stevia rebaudiana plant and compared it to spectra of paper, see Figure 7. As can be seen, the spectra show a relaxation peak at around $20 \mathrm{kHz}$ and this peak is absent in the spectra of the plant model. We conclude that we can get useful information about the plant with our set-up.

To further characterize the equipment and its adequacy for plant characterization, we measured a Stevia rebaudiana plant's leaf while increasing the temperature artificially in two different experimental designs (Figure 8 and Figure 9), the only difference between the two being the type of electrode configuration. Figure 8 shows the simultaneous measurement of temperature and relative humidity (RH), capacitance, loss and $\tan -\delta$ of a Stevia Stevia rebaudiana plant leaf and the same parameters for a piece of plastic, while full contact occurred between electrodes and sample. Figure 9, on the other hand, shows the same as Figure 8, but with only partial contact between electrodes and sample, as distance between electrodes is higher, and instead of plastic, air was measured as a reference. Although the distance between electrodes was adjustable, ensuring full contact between electrodes and sample implied that a bit of pressure was
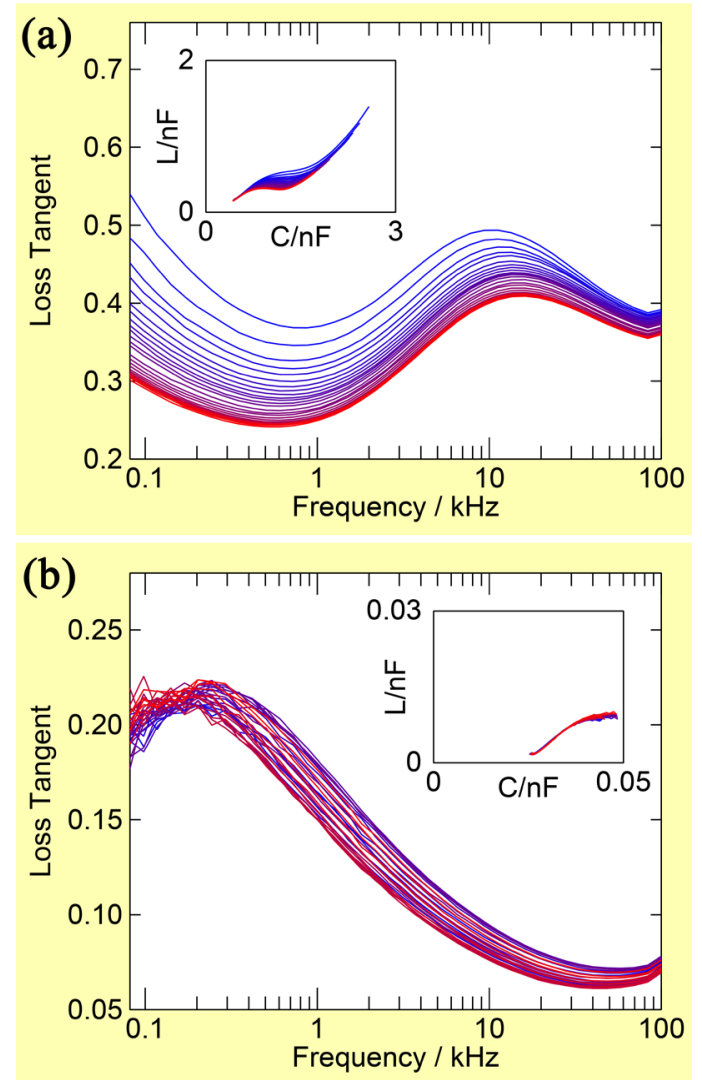

Figure 7. Loss-tangent (tan- $\delta=L / C$ ) spectra of Mentha spicata (a) and a dry paper sheet (b), note the different scales. The inset show Cole-Cole plots ( $L$ vs. $C$ ) of the same spectra. Spectra taken at 1 hour interval (blue is oldest, red is newest). A relaxation peak and low-frequency loss is seen in the plant spectra while the paper shows a relaxation peak at lower frequencies. 


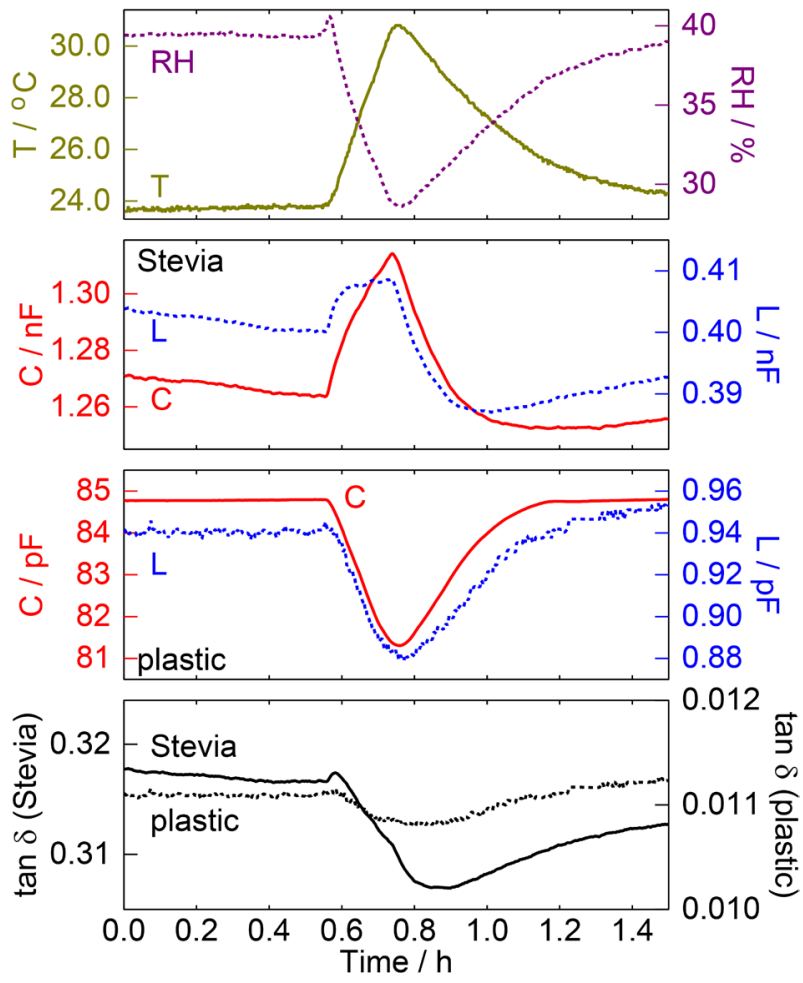

Figure 8. Effect of temperature and humidity. Temperature experiment with a Stevia rebaudiana leaf compared to a plastic sheet, fully contacted. A rapid rise in temperature (and accompanying decrease in relative humidity) increases the capacitance and loss of the plant, but lowers the same parameters of the plastic sheet. The loss tangent behaves similarly for both systems.

present, which is the case of Figure 8. In the case of Figure 9, as the distance between electrodes was higher, pressure on the leaf was inexistent, but there was only a partial contact between electrodes and sample, which proved to be inefficient in measuring the plant status, as it seemed to be measuring mostly the air between electrodes, more specifically, the resistivity and permittivity of air, thus, reflecting simply RH changes. When measuring the Stevia rebaudiana plant with full contact between electrodes and sample (leaf), we observed the increase of capacitance and loss with the increase of temperature and decrease of $\mathrm{RH}$, in direct contrast with something inert (in this case plastic, although paper behaves similarly, data now shown), in which capacitance and loss decreases.

When full contact existed between electrodes and sample (Figure 8), Stevia rebaudiana leaf's capacitance increased from $1.264 \mathrm{nF}$ to $1.313 \mathrm{nF}(3.87 \%)$ when temperature increased $6.6^{\circ} \mathrm{C}$ and $\mathrm{RH}$ decreased $11.9 \%$, thus capacitance increased $7.42 \mathrm{pF} /{ }^{\circ} \mathrm{C}$ and $4.12 \mathrm{pF} / \% \mathrm{RH}$. Stevia's leaf loss increased from $0.399 \mathrm{nF}$ to $0.409 \mathrm{nF}(2.5 \%)$ in the same situation, thus, loss increased $1.52 \mathrm{pF} /{ }^{\circ} \mathrm{C}$ and 0.84 $\mathrm{pF} / \% \mathrm{RH}$. Stevia rebaudiana leaf's tan- $\delta$ decreased from 0.317 to 0.307 (3.15\%), thus, $\tan -\delta$ decreased 0.00152 for each $1^{\circ} \mathrm{C}$ increase and decreased 0.00084 for each $1 \% \mathrm{RH}$ decrease. The capacitance of the plastic decreased from $84.79 \mathrm{pF}$ to $81.3 \mathrm{pF}$ (4.12\%; $\left.0.53 \mathrm{pF} /{ }^{\circ} \mathrm{C} ; 0.29 \mathrm{pF} / \% \mathrm{RH}\right)$, loss decreased from $0.945 \mathrm{pF}$ to 0.88 


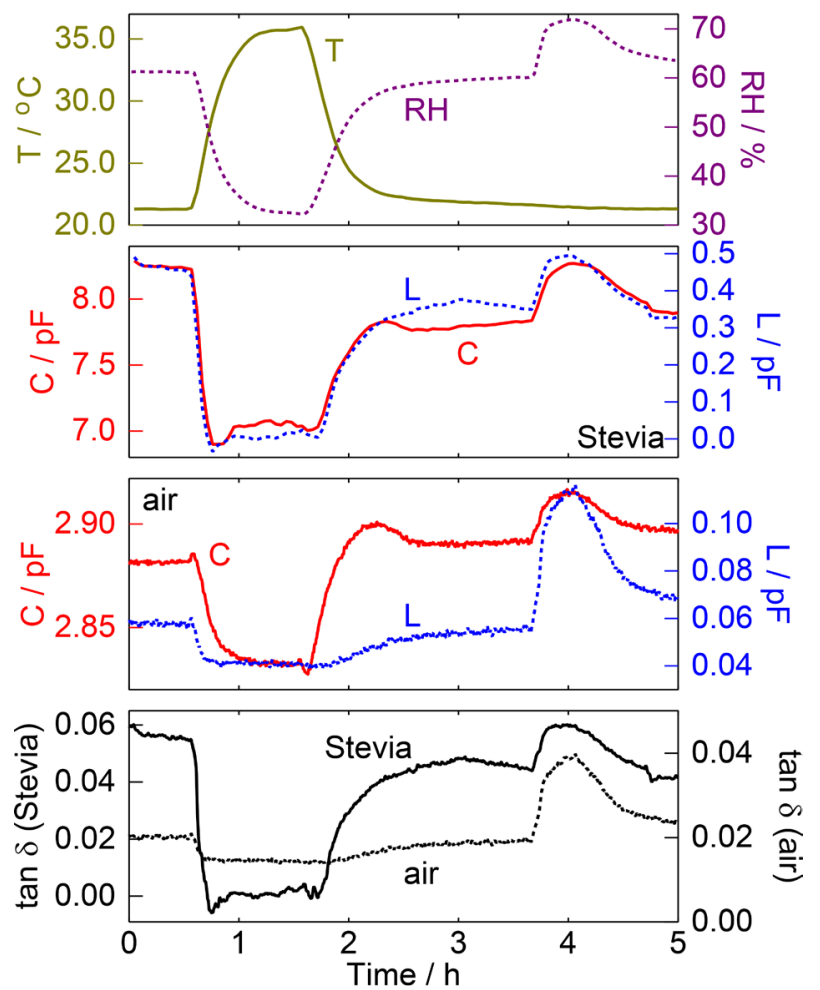

Figure 9. Effect of temperature and humidity. Temperature experiment with a Stevia rebaudiana leaf (partially contacted) compared to air. A rapid rise in temperature (and accompanying decrease in relative humidity) increases the capacitance and loss of both plant and air. The loss tangent behaves similarly for both systems. A later experiment changing only relative humidity.

$\mathrm{pF}\left(6.88 \% ; 0.0098 \mathrm{pF} /{ }^{\circ} \mathrm{C} ; 0.0055 \mathrm{pF} / \% \mathrm{RH}\right)$ and $\tan -\delta$ decreased from 0.0111 to $0.01078\left(2.9 \% ; 4.85 \times 10^{-5} /{ }^{\circ} \mathrm{C} ; 2.69 \times 10^{-5} / \% \mathrm{RH}\right)$.

In the case of partial contact between electrodes and sample (Figure 9), while temperature increased $14.8^{\circ} \mathrm{C}$ and $\mathrm{RH}$ decreased $29.1 \%$, Stevia rebaudiana leaf s capacitance decreased from $8.23 \mathrm{pF}$ to $6.86 \mathrm{pF}\left(16.65 \% ; 0.093 \mathrm{pF} /{ }^{\circ} \mathrm{C} ; 0.047\right.$ $\mathrm{pF} / \% \mathrm{RH}$ ), loss decreased from $0.45 \mathrm{pF}$ to approximately 0 (infinite resistance) $\left(100 \% ; 0.03 \mathrm{pF} /{ }^{\circ} \mathrm{C} ; 0.015 \mathrm{pF} / \% \mathrm{RH}\right)$ and $\tan -\delta$ decreased from 0.055 to approximately $0\left(100 \% ; 0.0037 /{ }^{\circ} \mathrm{C} ; 0.0019 / \% \mathrm{RH}\right)$. Air capacitance decreased from 2.88 $\mathrm{pF}$ to $2.83 \mathrm{pF}\left(1.74 \% ; 0.0034 \mathrm{pF} /{ }^{\circ} \mathrm{C} ; 0.0017 \mathrm{pF} / \% \mathrm{RH}\right)$, loss decreased from 0.06 $\mathrm{pF}$ to $0.04 \mathrm{pF}\left(33.3 \% ; 0.0014 \mathrm{pF} /{ }^{\circ} \mathrm{C} ; 0.0007 \mathrm{pF} / \% \mathrm{RH}\right)$ and $\tan -\delta$ decreased from 2 $\times 10^{-7}$ to $1.4 \times 10^{-7}\left(30 \% ; 4 \times 10^{-9} /{ }^{\circ} \mathrm{C} ; 2 \times 10^{-9} / \% \mathrm{RH}\right)$.

The fact that capacitance and loss of an inert material (as the plastic of Figure 8) in sudden temperature and humidity changes have an opposite behavior to a plant's respective parameters, suggests that capacitance/loss changes on the plant's measurement are not due (directly) to environmental factors, but actually represent the dynamics of the living plant.

Air humidity can be a significant factor in the measurements. When there is partial contact between sample and electrodes, capacitance and loss of the plant closely follow air humidity, suggesting that we are effectively just measuring the 
air. When there is full contact between sample and electrodes, when measuring a plant, although air humidity still plays a role, it is significantly smaller, sometimes almost imperceptible. Inert materials seem to behave similarly with full or partial contact with electrodes, following air humidity. This is in accordance with studies suggesting the use of capacitive sensors to measure air humidity [52]. We hypothesize that since paper or plastic have almost no water, it is more susceptible to outside factors. Plants, having significant water in normal conditions, provide a stronger signal, not being able to be influenced much by air humidity (except when all dried and dead, which then behave similarly to paper).

The fact that the behavior of plant and plastic/paper are completely opposite when heating occurs suggests that the capacitance/loss variations measured are not so much due to the thermal expansion of the measuring apparatus materials and/or changes in air humidity/permittivity. It is in any case useful to calculate the theoretical influence of thermal expansion and air humidity. In the introduction section we have derived the temperature dependence of the capacitance and loss (Equations (28) and (30)) and concluded that the areal expansion of the acrylic plates is dominating in our fixed-electrode system filled with air as used in the experiment of Figure 9, with the capacitance increasing and the loss decreasing by $140 \times 10^{-6}$ fraction per kelvin. The observed values were that the 2.88 $\mathrm{pF}$ decreased (rather than increased) $50 \mathrm{fF}$ in 14.8 kelvin $\left(1200 \times 10^{-6} / \mathrm{K}\right)$. The loss decreased dramatically $20 \mathrm{fF}$ from $60 \mathrm{fF}\left(300,000 \times 10^{-6} / \mathrm{K}\right)$. It is obvious that the behavior of this system cannot be explained by thermal effects. Temperature is rather irrelevant in the setup of fixed-distance "air-gapped" electrodes.

The drop in $\mathrm{RH}$ of $29.1 \%$ was accompanied by a $50 \mathrm{fF}$ drop in capacitance $\left(\beta_{\varepsilon}=600 \times 10^{-6} / \%\right)$ and a one-third drop in loss $\left(\beta_{\rho}=11000 \times 10^{-6} / \%\right)$, values far off the theoretical values as well. We conclude that our measured values are widely differing from the values of Choi [52], but that we do attribute the measured variations to changes in relative humidity rather than temperature, because the latter values are much better established and more amply documented in literature.

On the other hand, when using the clamped constant-pressure-electrodes configuration, as we have discussed in the introduction section, the humidity of air plays no part, and the capacitance is fully governed by the temperature dependence of $\varepsilon$ of water $\left(\alpha_{\varepsilon}=-4500 \times 10^{-6} / \mathrm{K}\right)$. However, our measurements show an increase of capacitance upon temperature, see Figure 8. Namely 7.42 pF in $6.6 \mathrm{~K}, \alpha_{\varepsilon}=900 \times 10^{-6} / \mathrm{K}$. Since the theoretical value of loss cannot be easily calculated (because it depends a lot on the exact composition of the plant material), we can only give an experimental value: $\beta_{\rho}=4000 \times 10^{-6} / \mathrm{K}$ and not compare it to theoretical calculations.

All effects here are much larger than expected on theoretical calculations and are even often of the wrong sign. We conclude that nature is much more complicated than simple theoretical ponderings.

Next, we proceeded to measure a plant (in this case a Mentha spicata) tran- 
sient behavior (admittance as a function of time), without watering it in order to induce water stress, until it died (Figure 10). Artificial light/dark cycles (chosen to be 12-hour periodic to not be equal to the natural circadian rhythm of the plant [58] [59] which can, by the way, also be disturbed [60]) clearly induce changes in the leaf's electrical properties, raising capacitance, loss and $\tan -\delta$ during the light periods and decreasing these during the dark periods. Also, the dying of the plant is clearly visible, with the sudden drop on all electrical parameters. After this point, watering to attempt rescuing the plant is no longer effective (data not shown; since the $\tan -\delta$ is here the ratio of zero divided by zero, it is effectively just noise). As control we tried to make a physical model, trying to simulate the plant (to confirm for example, that variations were not due simply to the soil getting moisture and we were not just observing variations of humidity and conductivity of the soil). This model consisted of a vase with soil and water, and an electrical wire connecting the soil to a piece of conductive paper. Then we measured this conductive paper, while simultaneously measuring the real plant, temperature and relative humidity $(\mathrm{RH})$. Capacitance, loss and $\tan -\delta$ of the plant do not correlate with temperature and RH changes and neither with the plant model (see the blue trace in the figure), demonstrating that during the measurements of a watered plant, the influence of $\mathrm{RH} /$ temperature factors on the measurements can be ignored. It is known that water is required for photosynthesis and also that, when lacking water, the stomata of the leaves are closed, thus avoiding losing further more water, but also inhibiting photosynthesis. Since the only variation in the plant system throughout time is loss of water due to evaporation and that our measurement technique is especially sensitive to water (due to its high dielectric constant), we can assume that the variations in light/dark electrical parameters are due to losing water. The effects of water loss on electrical properties are a well-known effect [34] [35] [47] [48].

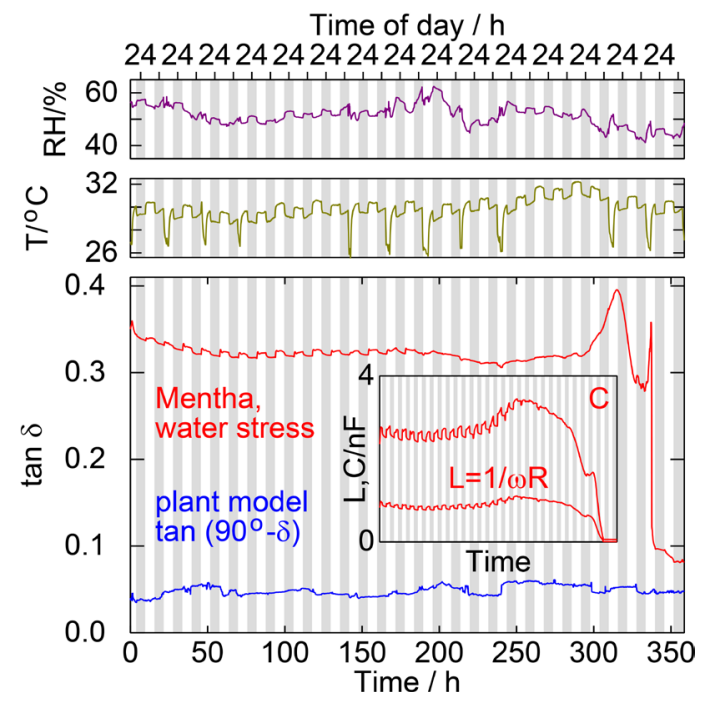

Figure 10. Admittance transients of Mentha spicata. Transient behavior of the loss tangent upon water stress. 
As discussed in the introduction, admittance signals can also be represented in the form of a vector on a complex plane in order to facilitate its analysis. It is formed by two components, $Y=G+j B$ : susceptance $(B=\omega C)$ and conductance ( $G=1 / R)$, see Equation (3). Both have the same unit (siemens, $S$ ) so that a magnitude $|Y|$ and a phase angle $\theta$ can be determined.

We formulated the following hypothesis: would it be possible to distinguish between different plants-ideally, the idea is to distinguish between sick and healthy plants of the same species-and other samples/materials through this admittance data, when represented in the complex plane? We represented our measurements in this way throughout time, but considering the high dynamic range of the data, we present them in logarithmic scales (Figure 11). These measurements had the length of several days, in which we submitted the plants to water stress, resulting in their deaths. We can observe that during the water stress, the plant signals migrate to the region where the signal of a normal piece of paper resides. Although apparently obvious-paper being processed cellulose, and cellulose being one of the major dry-matter constituents of a plant-it is nevertheless curious and intriguing, the fact that, electrically, paper is similar to a dry plant's leaf. As a comparison, we included the measurement of a physical model of a plant. To confirm that our observations were not due simply to the physical and inert aspect of a plant (for example, signal oscillations due to soil absorbing or evaporating water), we tried to model it by having watered soil in a pot, connecting the soil to a piece of conductive paper through an electrical wire, and measuring this piece of paper. No signal migration occurs when measuring the plant model, which suggests that the plant's signal migration is not due to pot water evaporation as this evaporation also occurred in the plant model measurement. Although this technique seems to allow easy distinction of

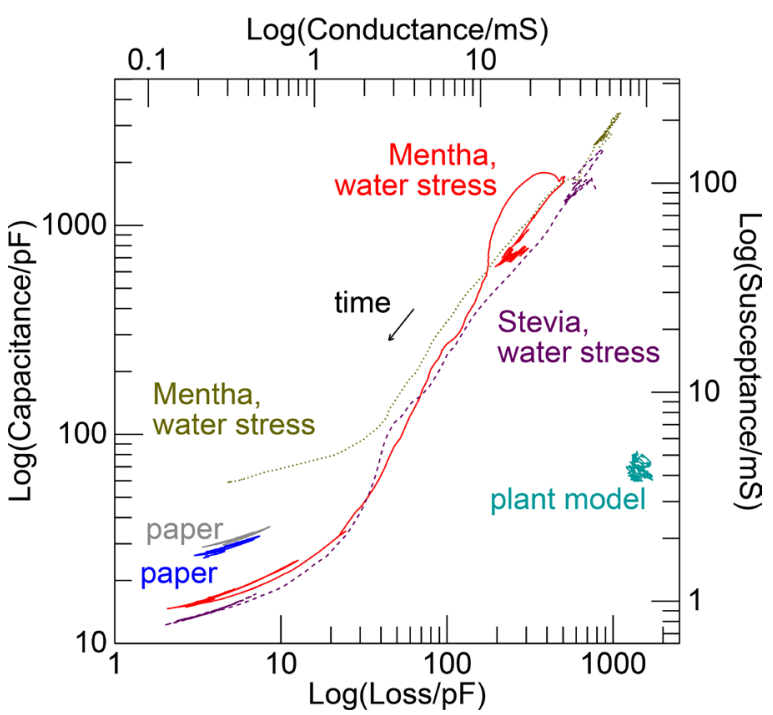

Figure 11. Fingerprint admittance transients of two Mentha spicata (red and green) and one Stevia rebaudiana (purple) plants undergoing water stress, as well as two normal paper sheets (blue and gray) and a physical plant model (cyan). 
plants from inert materials, distinction between plants seems more difficult as measurements are often subjective and relative to various factors such as plant age and size or leaf age, size and thickness, or the waxiness of the leaf surface.

\section{Conclusions}

Although there are limitations to this technique, such as being particularly sensitive to changes in relative humidity and being vulnerable to outside conditions such as the sun, wind or rain, our measurements show that it is possible to systematically obtain plant water status information from its electrical parameters when environmental conditions are minimally controlled as when in a greenhouse, using relatively cheap and off the shelf equipment as a lock-in amplifier. This could help the new technological agricultural paradigm where plant information is integrated in an Internet of Things concept and where irrigations occur automatically and at the optimum moment and with optimum quantity as to guarantee that the plant receives the amount of water needed while keeping water waste to a minimum, finally leading to a decrease in production costs and less ecological footprint.

We have shown that temperature is not very relevant for the measurements, but that relative humidity of the air is important, especially when contactless measurements are done. Partly because of this, we recommend the contacted "constant pressure" method. We also conclude that in-situ measurements remain difficult because of interference of the environment, specifically the strength of coupling of the plant to the rest of the world.

It has to be pointed out that the main strength of this set-up is to be able to determine the aqueous status of the plants. The more ambitious goal of being able to determine the health of a plant in terms of illnesses remains rather elusive.

\section{Acknowledgements}

The presented work was supported by project UID/Multi/00631/2013 (CEOT, Centro de Eletrónica, Optoelectrónica e Telecomunicações), funded by the Fundação para a Ciência e a Tecnologia (FCT) and the Ministério da Educação e Ciência (MEC), Portugal.

\section{Conflicts of Interest}

The authors declare no conflicts of interest regarding the publication of this paper.

\section{References}

[1] Berman, S., et al. (2012) Water Saving Potential in Agriculture in Europe: Findings from the Existing Studies and Application to Case Studies. Final Report Prepared for European Commission DG ENV.

[2] Gleick, P.H. (1993) Water in Crisis. Oxford University Press, New York. 
[3] Hasted, J.B. (1976) Aqueous Dielectrics. Vol. VII, 302 S. 112 Abb. 31 Tab. Series: Studies in Chemical Physics. Chapman and Hall, London.

[4] Elliott, S.R. (1998) The Physics and Chemistry of Solids. John Wiley \& Sons, Chichester.

[5] Lynden-Bell, R.M., et al. (2010) Water and Life: The Unique Properties of $\mathrm{H}_{2} \mathrm{O}$. CRC Press, Boca Raton. https://doi.org/10.1201/EBK1439803561

[6] Zhang, M.I.N. and Willison, J.H.M. (1993) Electrical Impedance Analysis in Plant Tissues: Impedance Measurement in Leaves. Journal of Experimental Botany, 44, 1369-1375. https://doi.org/10.1093/jxb/44.8.1369

[7] Stahlberg, R. (2006) Historical Overview on Plant Neurobiology. Plant Signaling \& Behavior, 1, 6-8. https://doi.org/10.4161/psb.1.1.2278

[8] Yan, X.F., et al. (2009) Research Progress on Electrical Signals in Higher Plants. Progress in Natural Science, 19, 531-541. https://doi.org/10.1016/j.pnsc.2008.08.009

[9] Foster, K.R. and Schwan, H.P. (1996) Dielectric Properties of Tissues. In: Polk, C. and Postow, E., Eds., Handbook of Biological Effects of Electromagnetic Fields, 2nd Edition, CRC Press, Boca Raton, 25-102.

[10] Miklavcic, D., Pavselj, N. and Hart, F.X. (2006) Electric Properties of Tissues. In: Akay, M., Ed., Encyclopedia of Biomedical Engineering, Wiley-Interscience, Hoboken, 1-12. https://doi.org/10.1002/9780471740360.ebs0403

[11] Cole, K.S. and Curtis, H.J. (1938) Electric Impedance of Nitella during Activity. Journal of General Physiology, 22, 37-64. https://doi.org/10.1085/igp.22.1.37

[12] Cole, K.S. and Curtis, H.J. (1939) Electric Impedance of the Squid Giant Axon during Activity. Journal of General Physiology, 22, 649-670. https://doi.org/10.1085/jgp.22.5.649

[13] Cole, K.S. and Curtis, H.J. (1936) Electric Impedance of Nerve and Muscle. Cold Spring Harbor Symposia on Quantitative Biology, 4, 73-89. https://doi.org/10.1101/SQB.1936.004.01.010

[14] Cole, K.S. (1928) Electric Impedance of Suspensions of Spheres. Journal of General Physiology, 12, 29. https://doi.org/10.1085/igp.12.1.29

[15] Cole, K.S. (1932) Electric Phase Angle of Cell Membranes. Journal of General Physiology, 15, 641. https://doi.org/10.1085/jgp.15.6.641

[16] Cole, K.S. (1941) Impedance of Single Cells. Tabulae Biologieae (Cellula, Pt. 2), 19, 24-27.

[17] Cole, K.S. and Hodgkin, A.L. (1939) Membrane and Protoplasm Resistance in the Squid Giant Axon. Journal of General Physiology, 22, 671. https://doi.org/10.1085/jgp.22.5.671

[18] Curtis, H.J. and Cole, K.S. (1937) Transverse Electric Impedance of Nitella. Journal of General Physiology, 21, 189. https://doi.org/10.1085/jgp.21.2.189

[19] Curtis, H.J. and Cole, K.S. (1938) Transverse Electric Impedance of the Squid Giant Axon. Journal of General Physiology, 21, 757. https://doi.org/10.1085/igp.21.6.757

[20] Greenham, C.G., Randall, P.J. and Müller, W.J. (1982) Studies of Phosphorus and Potassium Deficiencies in Trifolium subterraneum Based on Electrical Measurements. Canadian Journal of Botany, 60, 634-644. https://doi.org/10.1139/b82-084

[21] Muñoz-Huerta, R.F., et al. (2014) An Analysis of Electrical Impedance Measurements Applied for Plant N Status Estimation in Lettuce (Lactuca sativa). Sensors, 14, 11492-11503. https://doi.org/10.3390/s140711492 
[22] Tomkiewicz, D. and Piskier, T. (2012) A Plant Based Sensing Method for Nutrition Stress Monitoring. Precision Agriculture, 13, 370-383. https://doi.org/10.1007/s11119-011-9252-3

[23] Jackson, P.J. and Harker, F.R. (1999) Apple Bruise Detection by Electrical Impedance Measurement. HortScience, 35, 104-107. https://doi.org/10.21273/HORTSCI.35.1.104

[24] Cao, Y., et al. (2011) Analysis of the Willow Root System by Electrical Impedance Spectroscopy. Journal of Experimental Botany, 62, 351-358. https://doi.org/10.1093/jxb/erq276

[25] Jones, C.L., et al. (2006) Plant Biomass Estimation Using Dielectric Properties. Meeting Presentation (Portland), 063092.

[26] Postic, F. and Doussan, C. (2016) Benchmarking Electrical Methods for Rapid Estimation of Root Biomass. Plant Methods, 12, 1. https://doi.org/10.1186/s13007-016-0133-7

[27] van Emmerik, T., et al. (2017) Dielectric Response of Corn Leaves to Water Stress. IEEE Geoscience and Remote Sensing Letters, 14, 8-12. https://doi.org/10.1109/LGRS.2016.2606662

[28] van Emmerik, T., et al. (2015) A Comparison between Leaf Dielectric Properties of Stressed and Unstressed Tomato Plants. IEEE International Geoscience and Remote Sensing Symposium, Milan, 26-31 July 2015, 275-278. https://doi.org/10.1109/IGARSS.2015.7325753

[29] Sinha, K. and Tabib-Azar, M. (2016) Effect of Light and Water on Schefflera Plant Electrical Properties. Journal of Scientific Research \& Reports, 9, 1-11. https://doi.org/10.9734/JSRR/2016/19174

[30] He, J.-X., et al. (2011) A Prototype Portable System for Bioelectrical Impedance Spectroscopy. Sensor Letters, 9, 1151-1156. https://doi.org/10.1166/sl.2011.1384

[31] Afzal, A., Mousavi, S.F. and Khademi, M. (2010) Estimation of Leaf Moisture Content by Measuring the Capacitance. Journal of Agricultural Science and Technology, 12, 339-346.

[32] Afzal, A. and Mousavi, S.F. (2008) Estimation of Moisture in Maize Leaf by Measuring Leaf Dielectric Constant. International Journal of Agriculture and Biology, 10, 66-68.

[33] Jördens, C., et al. (2009) Evaluation of Leaf Water Status by Means of Permittivity at Terahertz Frequencies. Journal of Biological Physics, 35, 255-264. https://doi.org/10.1007/s10867-009-9161-0

[34] Burke, E.J., Harlow, R.C. and Ferré, P.A. (2005) Measuring the Dielectric Permittivity of a Plant Canopy and Its Response to Changes in Plant Water Status: An Application of Impulse Time Domain Transmission. Plant and Soil, 268, 123-133. https://doi.org/10.1007/s11104-004-0303-7

[35] Chuah, H.T., Kam, S.W. and Chye, Y.H. (1997) Microwave Dielectric Properties of Rubber and Oil Palm Leaf Samples: Measurement and Modelling. International Journal of Remote Sensing, 18, 2623-2639. https://doi.org/10.1080/014311697217503

[36] Greenham, C.G., Norris, D.O., Brock, R.D. and Thompson, A.M. (1952) Some Electrical Differences between Healthy and Virus-Infected Potato Tubers. Nature, 169, 973-974. https://doi.org/10.1038/169973a0

[37] Greenham, C.G., Helms, K. and Müller, W.J. (1978) Influence of Virus Infections on Impedance Parameters. Journal of Experimental Botany, 29, 867-877. 
https://doi.org/10.1093/jxb/29.4.867

[38] Borges, E., et al. (2012) Early Detection and Monitoring of Plant Diseases by Bioelectric Impedance Spectroscopy. IEEE 2nd Portuguese Meeting in Bioengineering, Coimbra, 23-25 February 2012, 1-4.

https://www.researchgate.net/publication/275539532

https://doi.org/10.1109/ENBENG.2012.6331377

[39] Dadshani, S., et al. (2015) Non-Invasive Assessment of Leaf Water Status Using a Dual-Mode Microwave Resonator. Plant Methods, 11, 8. https://doi.org/10.1186/s13007-015-0054-x

[40] Dietrich, R.C., et al. (2013) Can Root Electrical Capacitance Be Used to Predict Root Mass in Soil? Annals of Botany, 112, 457-464. https://doi.org/10.1093/aob/mct044

[41] Khalil, S.F., Mohktar, M.S. and Ibrahim, F. (2014) The Theory and Fundamentals of Bioimpedance Analysis in Clinical Status Monitoring and Diagnosis of Diseases. Sensors, 14, 10895-10928. https://doi.org/10.3390/s140610895

[42] Martinez, M.M. (2012) Utilização de Bioimpedâncianadeteção de árvoresinfetadas comescolítideos (Tomicusdestruens Wollaston) e com nemátode (Bursaphelenchusxylophilus. Nickel) da madeira de pinheiro (Portuguese). Thesis (Licenciatura) Universidade de Coimbra.

[43] Chatterjee, S.K., et al. (2015) Exploring Strategies for Classification of External Stimuli Using Statistical Features of the Plant Electrical Response. Journal of the Royal Society Interface, 12, Article ID: 20141225. https://doi.org/10.1098/rsif.2014.1225

[44] Christman, A. and Grill, E. (2013) Electric Defence. Nature, 500, 404-405. https://doi.org/10.1038/500404a

[45] Fromm, J. and Lautner, S. (2007) Electrical Signals and Their Physiological Significance in Plants. Plant, Cell and Environment, 30, 249-257. https://doi.org/10.1111/j.1365-3040.2006.01614.x

[46] Mousavi, S.A.R., Nguyen, C.T., Farmer, E.E. and Kellenberger, S. (2014) Measuring Surface Potential Changes on Leaves. Nature Protocols, 9, 1997-2004. https://doi.org/10.1038/nprot.2014.136

[47] Oyarce, P. and Gurovich, L. (2010) Electrical Signals in Avocado Trees Responses to Light and Water Availability Conditions Plant Signaling \& Behavior, 5, 34-41. https://doi.org/10.4161/psb.5.1.10157

[48] Ríos-Rojas, L., Tapia, F. and Gurovich, L.A. (2014) Electrophysiological Assessment of Water Stress in Fruit-Bearing Woody Plants. Journal of Plant Physiology, 171, 799-806. https://doi.org/10.1016/j.jplph.2014.02.005

[49] Volkov, A.G. (2012) Plant Electrophysiology: Methods and Cell Electrophysiology. Springer, Berlin. https://doi.org/10.1007/978-3-642-29119-7

[50] Davies, E. (2006) Plant Electrophysiology: Methods and Cell Electrophysiology. In: Volkov, A.G., Ed., Electrical Signals in Plants. Facts and Hypotheses, Springer, Berlin, 407-422. https://doi.org/10.1007/978-3-540-37843-3 17

[51] Stallinga, P. (2016) Electronic Instrumentation. Stallinga.org, Faro.

[52] Choi, J.M. and Kim, T.W. (2013) Humidity Sensor Using an Air Capacitor. Transactions on Electrical and Electronic Materials, 14, 182-186.

https://doi.org/10.4313/TEEM.2013.14.4.182

[53] The Engineering Toolbox. https://www.engineeringtoolbox.com/linear-expansion-coefficients-d 95.html

[54] Wikiepdia. Wikipedia: Thermal Expansion. 
https://en.wikipedia.org/wiki/Thermal expansion\\#Thermal expansion coefficient s for various materials

[55] Tomasanis, D. (1990) Effective Dielectric Constants of Foliage Media. ARCON Corporation Interim Report RADC-TR-90-157.

[56] Malmberg, C.G. and Maryott, A.A. (1956) Dielectric Constant of Water from $0{ }^{\circ} \mathrm{C}$ to $100{ }^{\circ} \mathrm{C}$. Journal of Research of the National Bureau of Standards, 56, 2641. https://doi.org/10.6028/jres.056.001

[57] Pawar, S.D., Murugavel, P. and Lal, D.M. (2009) Effect of Relative Humidity and Sea Level Pressure on Electrical Conductivity of Air over Indian Ocean. Journal of Geophysical Research, 114, D02205. https://doi.org/10.1029/2007JD009716

[58] McClung, C.R. (2006) Plant Circadian Rhythms. The Plant Cell, 18, 792-803. https://doi.org/10.1105/tpc.106.040980

[59] Volkov, A.G., et al. (2012) Circadian Rhythms in Biologically Closed Electrical Circuits of Plants. Plant Signaling \& Behavior, 7, 282-284. https://doi.org/10.4161/psb.18798

[60] Fukuda, H., Murase, H. and Tokuda, I.T. (2013) Controlling Circadian Rhythms by Dark-Pulse Perturbations in Arabidopsis thaliana. Scientific Reports, 3, 1533. https://doi.org/10.1038/srep01533 